\title{
Techniques of Computing Optimization for Satellite Monitoring of Sea Surface
}

\author{
Alexander B. Murynin ${ }^{1,2}$, Karen S. Khachatran ${ }^{1}$ \\ ${ }^{1}$ Institute for Scientific Research of Aerospace Monitoring "AEROCOSMOS", Moscow, Russia \\ office@aerocosmos.info \\ ${ }^{2}$ Federal Research Center "Informatics and Control" of the Russian Academy of Sciences, Moscow, \\ Russia \\ frccsc@frccsc.ru
}

\begin{abstract}
High-performance methods are described for recording the spectra of the sea surface from space images for solving problems of operational oceanography. Algorithms have been developed that implement methods for quickly reconstructing the characteristics of the atmosphere-hydrosphere interface using satellite imagery and research software for quickly reconstructing the characteristics of the sea surface from satellite imagery. The developed algorithms were tested and their performance was estimated using experimental data. The developed prototype of research software is designed to work with the use of multi-core processors. The program performs the restoration of the spectra of slopes and elevations of the sea surface using the developed high-performance methods. The performed computational experiments showed the feasibility of increasing the productivity of recording the spectra of slopes and elevations of the sea surface from the spectra of space images due to parallelization of calculations.
\end{abstract}

Keywords: remote sensing, sea surface waves, wave spectra, satellite image processing

Copyright (C2019 by the paper's authors. Copying permitted for private and academic purposes 


\title{
МЕТОДЫ ОПТИМИЗАЦИИ ВЫЧИСЛЕНИЙ ПРИ КОСМИЧЕСКОМ МОНИТОРИНГЕ МОРСКОЙ ПОВЕРХНОСТИ
}

\author{
А.Б. Мурынин ${ }^{1,2}$, К.С. Хачатрян ${ }^{1}$ \\ ${ }^{1}$ Научно-исследовательский институт аэрокосмического мониторинга "АЭРОКОСМОС", \\ Москва, Россия \\ office@aerocosmos.info \\ ${ }^{2}$ Федеральный исследовательский центр «Информатика и управление» Российской Академии \\ Наук, Москва, Россия \\ frccsc@frccsc.ru
}

Описаны высокопроизводительные методы регистрации спектров морской поверхности по космическим изображениям для решения задач оперативной океанографии. Разработаны алгоритмы, реализующие методы оперативного восстановления характеристик границы раздела атмосфера-гидросфера по космическим изображениям и исследовательское программное обеспечение для оперативного восстановления характеристик морской поверхности по космическим изображениям. Проведено тестирование разработанных алгоритмов и получены оценки их производительности на экспериментальных данных. Разработанный прототип исследовательского программного обеспечения предназначен для работы с применением многоядерных процессоров. Программа выполняет восстановление спектров уклонов и возвышений морской поверхности с применением разработанных высокопроизводительных методов. Проведённые вычислительные эксперименты показали целесообразность увеличения производительности регистрации спектров уклонов и возвышений морской поверхности по спектрам космических изображений за счёт распараллеливания вычислений.

Ключевые слова: дистанционное зондирование, взволнованная морская поверхность, спектры волнения, обработка космических изображений

\section{Введение}

Целью работы является разработка высокопроизводительных методов регистрации спектров морской поверхности по космическим изображениям для решения задач оперативной океанографии, в интересах охраны окружающей среды и рационального природопользования акваторий морей и океанов.

Регистрация спектров поверхностного волнения является актуальной при решении многих фундаментальных и прикладных задач современной оперативной океанологии. Получение информации о таких спектрах важно для исследования различных физических процессов, происходящих вблизи границы раздела океан-атмосфера, а также для оперативного обнаружения загрязнений водной среды и мониторинга антропогенных воздействий на морские акватории. Для получения двумерных спектров поверхностного волнения на больших площадях, целесообразно использование методов дистанционного зондирования, основанных на обработке космических изображений высокого пространственного разрешения, а также изображений, получаемых с воздушных носителей. Адекватная оценка спектров морской поверхности, формируемых путем обработки изображений, получаемых в процессе аэрокосмического мониторинга акваторий, требует использования восстанавливающих операторов, 
представляющих собой функции, позволяющие преобразовать поля яркости, регистрируемые на оптических аэрокосмических изображениях, в спектры морского волнения. Эти операторы строятся с использованием методов численного моделирования на основе учета различных условий формирования аэрокосмических изображений и характеристик аппаратуры дистанционного зондирования.

В связи с необходимостью обработки больших объемов данных дистанционного зондирования обширных акваторий актуальна разработка высокопроизводительных методов и алгоритмов восстановления спектров поверхностного волнения по оптическим изображениям высокого пространственного разрешения. Такие методы и алгоритмы основаны на выполнении процедур восстановления спектров уклонов и возвышений морской поверхности с применением нелинейных восстанавливающих операторов с использованием параллельных вычислительных потоков.

В качестве базового метода для разработки высокопроизводительных алгоритмов регистрации спектров морской поверхности по космическим изображениям для решения задач оперативной океанографии используется нелинейный метод восстановления спектров морской поверхности [1-6].

В настоящей работе мы приводим некоторые результаты по оптимизации скорости обработки космических данных и по применению автоматизации при обработке космических данных

\section{Оптимизация скорости обработки космических данных}

При анализе пространственно-неоднородного волнения целесообразно разделить изображение на фрагменты, имеющие размер, соответствующий характерному масштабу изучаемых явлений. Стандартным подходом является разбиение изображения на равные квадратные фрагменты (тайлы) с возможностью их частичного перекрытия. Пример обработки с таким разбиением приведен ниже.

Разбиение на фрагменты (тайлы) позволяет производить обработку этих тайлов параллельно при наличии такой возможности. Алгоритм параллельной обработки тайлов кратко может быть описан в виде следующей последовательности действий.

1) Открыть изображение и считать сопроводительную информацию.

2) Считать тайл изображения заданного размера из заданного места на исходном изображении и определить параметры условий формирования поля яркости в этом тайле, влияющие на параметры нелинейного восстанавливающего оператора [3,5];

3) Применить преобразование Фурье к фрагменту изображения и вычислить двумерную пространственную спектральную плотность фрагменту изображения, которую далее для краткости будем называть просто спектром тайла изображения.

4) Получить нелинейный восстанавливающий оператор в виде пространтвенно-частотного фильтра $R(\boldsymbol{k})$, зависящего от условий формирования изображения [3-5]:

$$
R(\boldsymbol{k})=a_{0}\left(\exp \left(a_{4} k^{a_{5}}\right)\right)\left(\left(\cos \left(\varphi-\varphi_{c}\right)\right)^{a_{3}} k^{a_{1}+a_{2} \cos \left(\varphi-\varphi_{c}\right)}\right.
$$

где

$\boldsymbol{k}$ - волновой вектор;

$\boldsymbol{a}=\left(a_{0}, a_{1}, a_{2}, a_{3}, a_{4}, a_{5}\right)$ - вектор параметров аппроксимации, зависящих от условий формирования поля яркости морской поверхности,

Компоненты вектора имеют различный физический смысл.

Компоненты области степенного спадания спектральной плотности волнения [4,5]:

$a_{1}$ - компенсирует нелинейные искажения показателя степенной функции спектра волнения для направления $\varphi_{\mathrm{C}}$, определяемого азимутом Солнца;

$a_{2}$ - определяет угловую зависимость показателя степенной функции спектра волнения;

$a_{3}$ - определяет угловую зависимость спектральной энергии. 
Компоненты $a_{4}, a_{5}$ описывают восстанавливающий фильтр в области низких пространственных частот [3].

5) Применить нелинейный восстанавливающий фильтр $R(\boldsymbol{k})$ к спектру тайла изображения морской поверхности и получить спектр уклонов морской поверхности:

$$
\Phi(\boldsymbol{k})=R(\boldsymbol{k}) S(\boldsymbol{k})
$$

6) Рассчитать характеристики спектра уклонов морской поверхности, в том числе параметр $p$ степенной аппроксимации

$$
\Phi(\boldsymbol{k}) \sim k^{-p}
$$

7) Сохранить полученные значения характеристик спектров в векторный файл в формате SHP (шейп-файл);

8) Вывести полученный шейп-файл

При этом шаги 2 - 6 описанного алгоритма производятся параллельно для всех имеющихся тайлов с использованием всех доступных ядер процессоров.

Блок схема алгоритма приведена на рисунке 1 .

Для тестирования разработанных алгоритмов использовались изображения высокого пространственного разрешени полученные аппаратурой спутника GeoЕуе в акватории Тихого океана [7-14]

\section{Программная реализация алгоритмов}

Для реализации описанного алгоритма разработан программный модуль на языке С++, позволяющий работать с форматами входных изображений TIFF, GeoTIFF. Параллельность вычисления реализуется с использованием спецификации OpenMP [15].

Программный модуль поддерживает многоядерные процессоры и содержит в себе оптимизированные функции для обработки мультимедийных данных.

При разработке модуля использованы библиотеки:

- OpenCV, библиотека алгоритмов компьютерного зрения, обработки изображений и численных алгоритмов общего назначения с открытым кодом, реализована на $\mathrm{C} / \mathrm{C}++[16]$;

- Intel IPP (Intel Integrated Performance Primitives), которая содержит наиболее эффективную реализацию быстрого преобразования Фурье (БПФ, FFT) для процессоров Intel, на которых и тестировался данный код [17];

- $\quad$ GDAL (Geospatial Data Abstraction Library) - библиотека для чтения и записи растровых и векторных гео-пространственных форматов данных, которая отвечает за работу с форматом GeoTIFF, который позволяет хранить изображения очень большего размера, считывать, записывать фрагменты изображения, работать с геопривязкой [18].

Код разработанного модуля имеет две реализации:

- Стандартную реализацию, работающую на стандартном центральном процессоре;

- $\quad$ Реализацию высокопроизводительного модуля регистрации спектров, основанную на использовании программно-аппаратной архитектуры параллельных вычислений CUDA c использованием графических процессоров [19].

Для оценки эффективности распараллеливания вычисления проводилось тестирование времени выполнения и вычислялись значения таких характеристик как ускорение и эффективность.

Ускорение есть отношение времени выполнения вычислений на одном потоке, на время выполнения тех же вычислений в многопотоковом режиме:

Где:

$$
S=\frac{T_{1}}{T_{P}}
$$

$T_{1}$ - время выполнения вычислений на одном потоке,

$T_{p}$ - время выполнения тех же вычисления на р потоках. 
Эффективность есть отношения ускорения к количеству потоков:

где $S$ ускорение на $p$ потоках.

$$
E=\frac{S}{p}
$$

Данные по ускорению и эффективности, полученные при обработке тестового изображения, приведены в таблице 1. Оценки получены при размере тайла 2048х2048 пикселов. Результаты исследования влияние размера тайла на время обработки тестового изображения приведены в таблице 2. Приведённые результаты позволяют заключить, что минимум времени исполнения наблюдается при размерах тайла 1024x1024 и 2048х2048. При уменьшении и при увеличении размера тайла наблюдается увеличение времени выполнения программы.

При автоматизированной обработке изображений большого размера могут возникать проблемы по дальнейшему анализу и представлению полученных данных.

Для тщательной обработки и анализа результатов необходимо удобное представление полученных массивов информации. Предлагаемым подходом является автоматическое представление результатов обработки изображений в формате презентации PowerPoint.

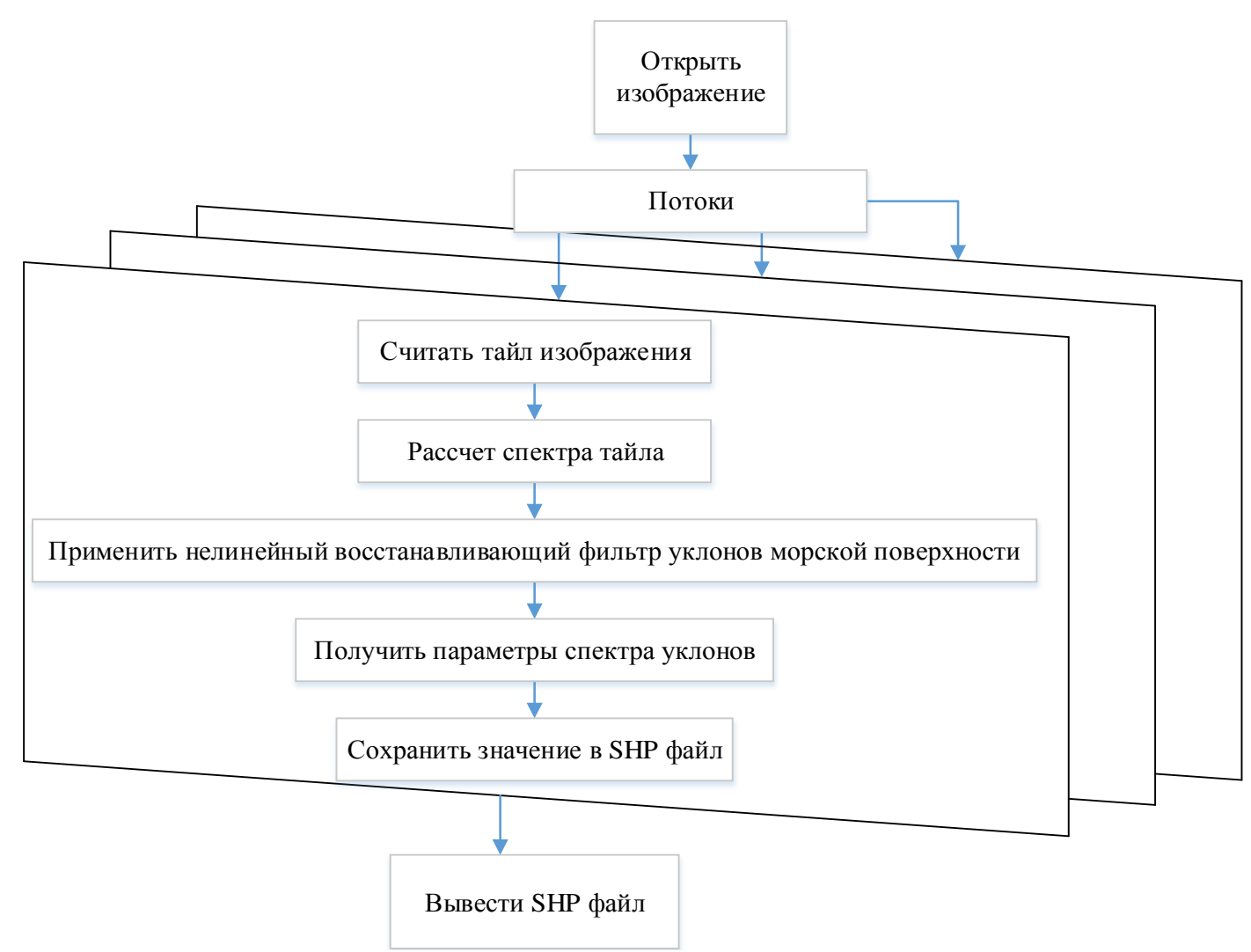

Рисунок 1. Блок схема алгоритма обработки космического изображения морской поверхности и регистрации пространственных спектров уклонов

С точки зрения изучения развития волнения на обширных акваториях представляет интерес формирование выходных данных презентации следующего состава:

- исходное изображение тайла;

- спектр изображения;

- спектр уклонов морской поверхности, восстановленный с применением нелинейного оператора;

- спектр уклонов морской поверхности, прошедший медианную фильтрацию изображения для лучшего визуального представления;

- график одномерного разреза спектра уклонов в заданном направлении, либо интегральный одномерный спектр уклонов в заданном диапазоне углов. 
Таблица 1. Значения ускорения и эффективности по тестовому изображению

\begin{tabular}{|c|c|c|c|}
\hline Количество потоков & $\begin{array}{c}\text { Время выполнения } \\
(\mathbf{c})\end{array}$ & Эффективность (\%) & Ускорение \\
\hline 1 & 195 & 100 & 1 \\
\hline 2 & 94 & 100 & 1.99 \\
\hline 4 & 51 & 95.5 & 3.82 \\
\hline 8 & 39 & 62.5 & 5 \\
\hline
\end{tabular}

Таблица 2. Значения времени выполнения для разных размеров тайла

\begin{tabular}{|c|c|}
\hline Размер одной стороны тайла (пикселов) & Время выполнения (c) \\
\hline 512 & 55 \\
\hline 1024 & 51 \\
\hline 2048 & 51 \\
\hline 4096 & 54 \\
\hline
\end{tabular}

Для построения презентаций большого объёма разработан скрипт на встроенном в PowerPoint языке Visual Basic. Пример фрагмента презентации проведён на рис. 2(a). Там же, на рис.2(б), приведён результат обработки тестового изображения в виде панорамы, представляющей пространственное распределение степенного показателя спектра волнения в тестовой акатории.

Image tile:
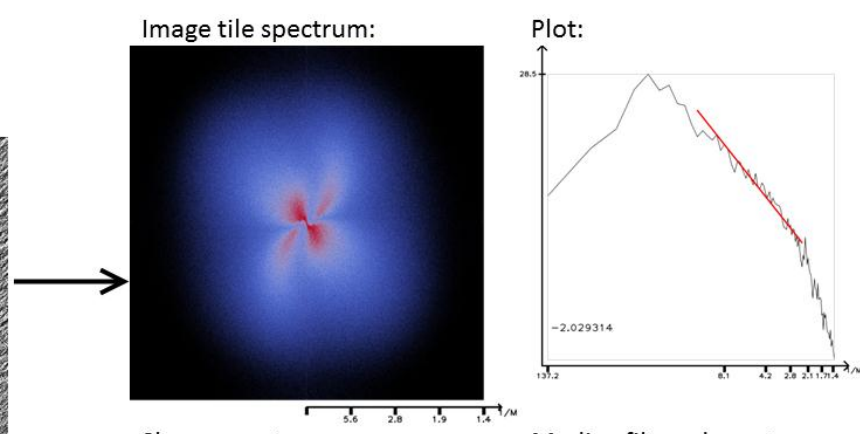

Slopes spectrum:

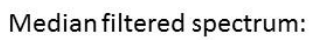

(a)
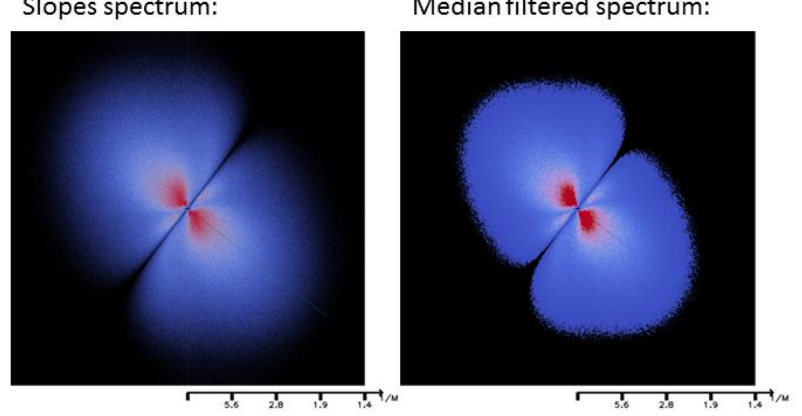

(b)

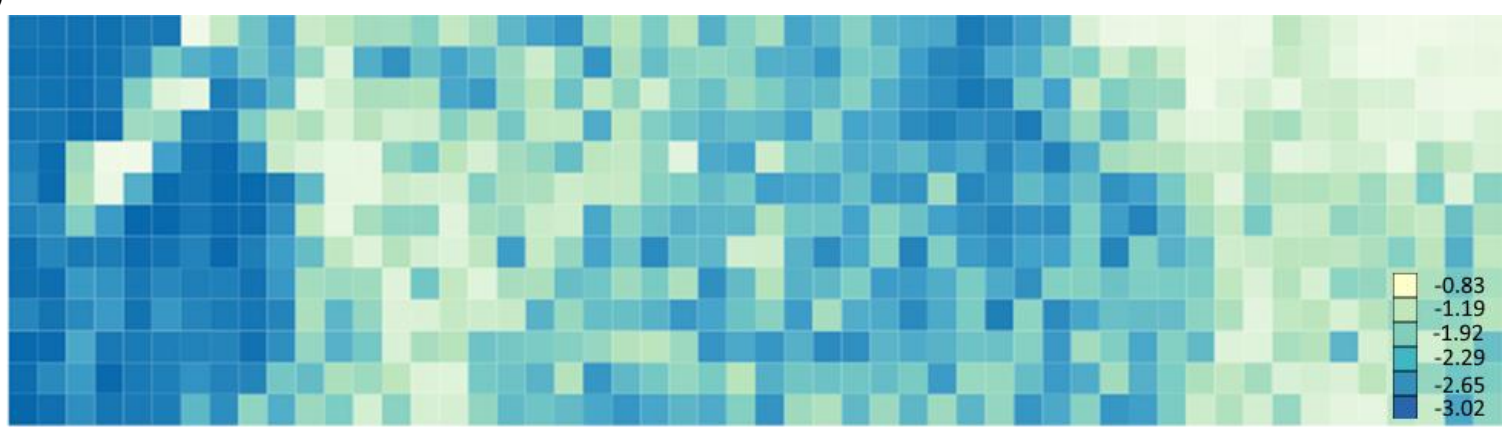

Рисунок 2. Пример обработки космического изображения в автоматическом режиме:

(a)- страница презентации с результатами обработки одного тайла размером 2048х2048 (Обрабатываемое изображение имеет размер 108294х28236 пикселов и разрядность 16bit.); (b) визуализация восстановленных степенных показателей пространственного спектра волнения. 


\section{Заключение}

Проведённые вычислительные эксперименты показывают целесообразность применения используемого подхода и разработанных алгоритмов для увеличения вычислительной производительности при решении задач регистрации спектров уклонов и возвышений морской поверхности по спектрам космических изображений.

Результаты работы могут быть использованы при создании программного обеспечения многопроцессорных аппаратно-программных комплексов, создаваемых для оперативного мониторинга процессов и явлений, происходящих в различных акваториях мирового океана по данным, поступающим с космических и авиационных носителей.

Работа поддержана Минобрнауки России, Государственное задание № 075-00896-19-00.

\section{References}

[1] Bondur V.G., Murynin A.B. Methods for retrieval of sea wave spectra from aerospace image spectra, Izvestiya, Atmospheric and Oceanic Physics, 2016, 52(9), pp. 877-887. DOI: 10.1134/S0001433816090085.

[2] Bondur V.G., Dulov V.A., Murynin A.B., Yurovsky Yu.Yu. A study of sea-wave spectra in a wide wavelength range from satellite and in-situ data, Izvestia, Atmospheric and Oceanic Physics, 2016, 52(9), pp. 888-903, DOI: 10.1134/S0001433816090097

[3] Bondur V.G., Dulov V.A., Murynin A.B., Ignatiev V.Yu. Retrieving sea-wave spectra using satellite-imagery spectra in a wide range of frequencies, Izvestia, Atmospheric and Oceanic Physics, 2016, 52(6), pp. 637-648.

[5] Murynin A.B., Restoration of the spatial spectra of the sea surface from optical images in a nonlinear model of the brightness field, Issled. Zemli Kosmosa, 1990, no. 6, pp. 60-70 (In Russian).

[5] Murynin A.B., Parameterization of filters retrieving the spatial spectra of sea surface slopes on the basis of optical imagery, Issled. Zemli Kosmosa, 1991, no. 5, pp. 31-38 (In Russian).

[6] Bondur V.G., Murynin, A.B., Reconstruction of the spectra of the surface waves from the spectra of their images with an account of the nonlinear modulation of the brightness field, Opt. Atmos. Okeana, 1991, vol. 4, no. 4, pp. 387-393 (In Russian).

[7] Bondur V.G. Satellite monitoring and mathematical modelling of deep runoff turbulent jets in coastal water areas, In book Waste Water - Evaluation and Management, ISBN 978-953-307-233-3, InTech, Croatia. 2011. P. 155-180. http:// www.intechopen.com/articles/show/title/satellite-monitoring-and-mathematical-modelling-ofdeep-runoff-turbulent-jets-in-coastal-water-areas.

[8] Bondur V. Complex Satellite Monitoring of Coastal Water Areas // 31st International Symposium on Remote Sensing of Environment. ISRSE, 2005. 7p.

[9] Bondur V, Tsidilina M. Features of Formation of Remote Sensing and Sea truth Databases for The Monitoring of Anthropogenic Impact on Ecosystems of Coastal Water Areas // 31st International Symposium on Remote Sensing of Environment. ISRSE, 2005, pp. 192-195.

[10] Keeler R., Bondur V., Vithanage D. Sea truth measurements for remote sensing of littoral water, Sea Technology, April, 2004. pp.53-58.

[11] Bondur V.G., Grebenyuk Yu.V., Sabinin K.D. Variability of internal tides in the coastal water area of Oahu Island (Hawaii), Oceanology, 2008, Vol. 48, No. 5, pp. 611-621. DOI:10.1134/S0001437008050019.

[12] Bondur V.G., Grebenyuk Yu.V., Sabinin K.D. The spectral characteristics and kinematics of short-period internal waves on the Hawaiian shelf, Izvestiya, Atmospheric and Oceanic Physics, 2009, Vol. 45, No. 5, pp. 598607. DOI: 10.1134/S0001433809050077

[13] Bondur V.G., Zhurbas V.M., Grebenyuk Yu.V. Mathematical Modeling of Turbulent Jets of Deep-Water Sewage Discharge into Coastal Basins, Oceanology, 2006, Vol.46, No.6, pp. 757-771. DOI: 10.1134/S0001437006060014.

[14] Bondur V.G. Aerospace Methods and Technologies for Monitoring Oil and Gas Areas and Facilities, Izvestiya, Atmospheric and Oceanic Physics, 2011, Vol. 47, No. 9, pp. 1007-1018. DOI: 10.1134/S0001433811090039. 
[15] The OpenMP API specification for parallel programming. Available at: https://www.openmp.org (accessed 06.03.2019)

[16] Open Source Computer Vision Library. Available at: https://opencv.org (accessed 06.03.2019)

[17] Intel Integrated Performance Primitives. Available at: https://software.intel.com/en-us/intel-ipp (accessed 06.03.2019)

[18] Geospatial Data Abstraction Library. Available at: https://www.gdal.org (accessed 06.03.2019)

[19]CUDA parallel computing. Available at: https://developer.nvidia.com/cuda-zone (accessed 06.03.2019) 